\title{
Inhomogeneous Winding for Loosely Coupled Transformers to Reduce Magnetic Loss
}

\author{
Jing Zhou $\mathbb{D}^{1,2,3}$ Jiazhong $\mathrm{He} \mathbb{D}^{2,3}$ and Fan Zhu $\mathbb{D}^{2}$ \\ ${ }^{1}$ Hainan Institute, Zhejiang University, Sanya 572025, China \\ ${ }^{2}$ College of Electrical Engineering, Zhejiang University, Hangzhou 310027, China \\ ${ }^{3}$ Polytechnic Institute, Zhejiang University, Hangzhou 310015, China \\ Correspondence should be addressed to Jing Zhou; jingzhou@zju.edu.cn
}

Received 25 June 2021; Accepted 14 September 2021; Published 5 October 2021

Academic Editor: Giuseppina Monti

Copyright ( $\odot 2021$ Jing Zhou et al. This is an open access article distributed under the Creative Commons Attribution License, which permits unrestricted use, distribution, and reproduction in any medium, provided the original work is properly cited.

Wireless power transfer has been proved promising in various applications. The homogeneous winding method in loosely coupled transformers incurs unnecessary intense magnetic field distribution in the center and causes extra magnetic loss. An inhomogeneous winding method is proposed in this paper, and a relatively homogeneous magnetic field distribution inside the core is achieved. This paper investigated the magnetic loss of homogeneous winding and inhomogeneous winding for wireless power transfer. A theoretical model was built to evaluate magnetic loss under inhomogeneous winding. The coupling coefficient and magnetic loss were investigated individually and comparisons were made between different width ratio combinations. Theoretical analysis was validated in experiments.

\section{Introduction}

Wireless power transfer eliminates the need for wires to connect the load from power source, and it has broad prospects in implantable medical devices, electric vehicles, etc. Several approaches to improve the energy efficiency of the wireless coupled coils have been developed [1-5].

Homogeneous winding, i.e., maintain the same distance between each turn, is widely applied in loosely coupled transformers [6-8]. And, this traditional winding method incurs the inhomogeneous internal magnetic field distribution; the magnetic induction intensity is concentrated in the central area, which results in greater loss in core center. An inhomogeneous winding method is proposed in this paper: coils were loosely winded in the center while tightly winded on two ends (Figure 1), so as to realize homogeneous magnetic field distribution inside the core (Figure 2) and reduce magnetic loss.

Steinmetz equation is the most used method to characterize core losses [9, 10]. However, for wireless power transfer system, the uneven flux density distribution in the core makes it difficult to employ Steinmetz equation directly.
Moreover, if we divide the core into several sections, the core loss in each section is incurred not only by its own windings but also by its adjacent windings. Alternatively, FEM simulation software is widely applied to calculate core loss $[11,12]$. However, this method is very time-consuming, especially for more accurate $3 \mathrm{D}$ models. In addition, the optimization of system parameters can only by realized by sweeping design parameters. The optimized point could be missed since it lacks an overall understanding of the whole optimization region. In the view of these problems, a magnetic circuit model [13] is proposed in this paper; it is valid for solenoid winding structure and convenient to obtain flux distribution.

It would be desirable to reduce the magnetic loss while maintaining tight and compact windings. In this paper, different winding parameters were investigated and compared, in terms of coupling coefficient and magnetic loss.

This paper is arranged as follows. A magnetic circuit model is proposed in Section 2 to calculate the magnetic loss under inhomogeneous winding. Section 3 investigates the influence of winding parameters. The experimental setup and results are discussed in Section 4. Conclusions are drawn in Section 5. 


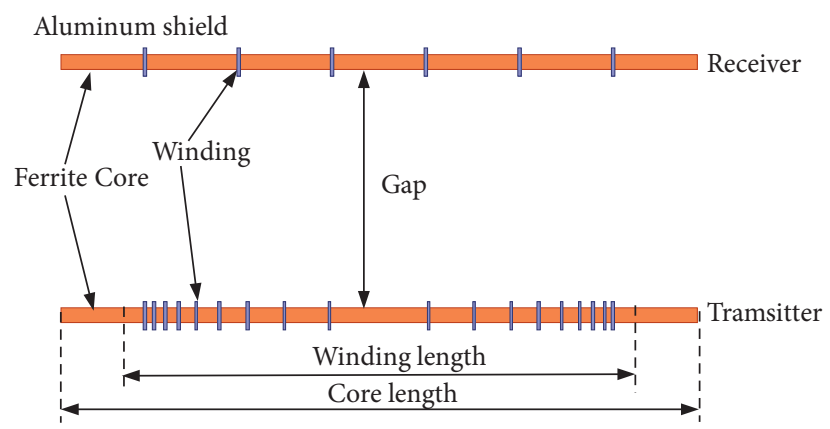

FIGURE 1: Structure of inhomogeneous winding for loosely coupled transformers.

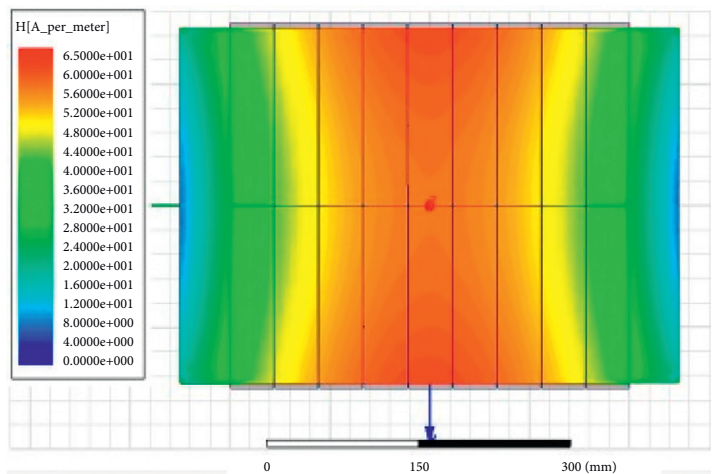

(a)

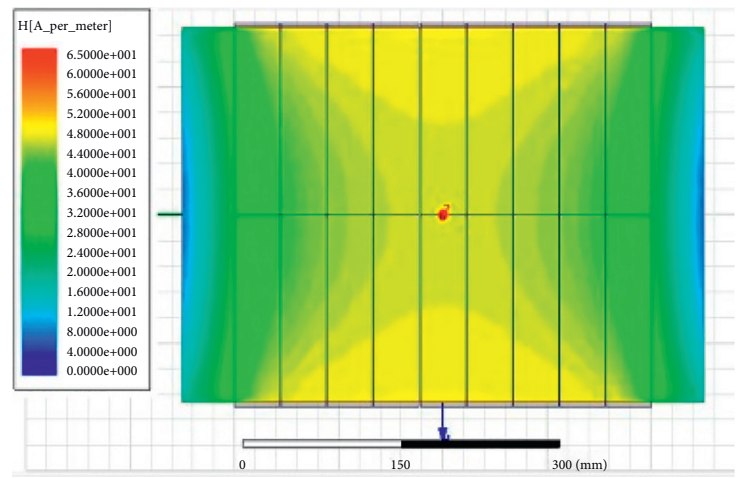

(b)

FIGURE 2: Internal magnetic field. (a) Homogeneous winding. (b) Inhomogeneous winding.

\section{Magnetic Losses of Ferrites under Inhomogeneous Winding}

A typical wireless power transfer system is illustrated in Figure 3. It contains a full-bridge inverter and rectifier and corresponding compensating topology. The loosely coupled transformer contains a transmitter coil, a receiver coil, and corresponding magnetic cores. In this paper, the transmitter and receiver coils are both solenoid winding. On the primary side, the resonant capacitor $C_{P}$ connects in series with the transmitter coil, to form the resonant network. On the secondary side, the resonant capacitor $C_{S}$ connects in parallel with the receiver coil.

A typical flux distribution of the solenoid structure is shown in Figure 4. The total flux concerns the internal leakage flux, external leakage flux, and mutual flux, among which the internal leakage flux comprises the majority of leakage flux since its path length is much shorter than others.

Corresponding equivalent magnetic reluctance network is analyzed in Figure 5. As seen in Figure 5, the core is divided into 7 parts in longitudinal direction. In order to clearly demonstrate the magnetic motive force and the magnetic reluctance in each flux path, at least 7 divisions have to be provided. With more divisions, theoretically, we can obtain a more accurate result, but the calculation complexity will increase dramatically. The discretization number is a trade-off between precision and complexity. Each core section is modelled as magnetic reluctance, while core section with excitation winding is modelled as a voltage

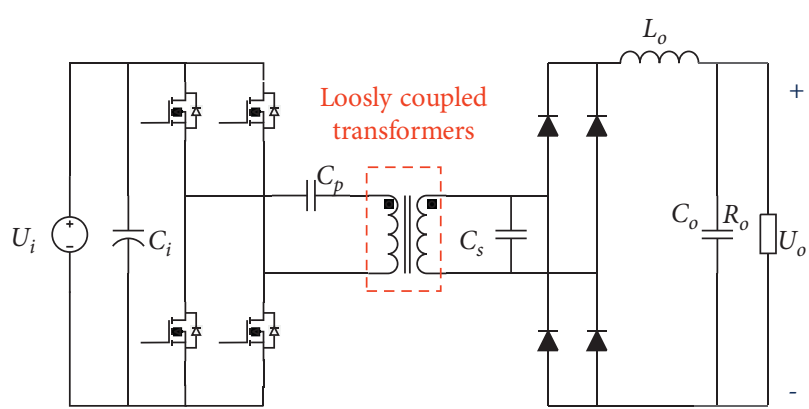

FIGURE 3: Typical wireless power transfer system.

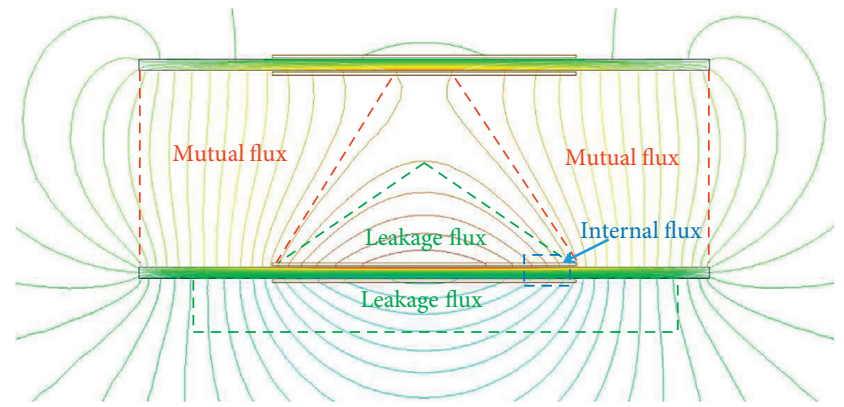

FIGURE 4: Flux distribution of the solenoid structure.

source in series with magnetic reluctance. The voltage source corresponds to the number of turns and current excitation in the winding, represented by $\alpha \cdot i_{p}$ in Figure 5 . 


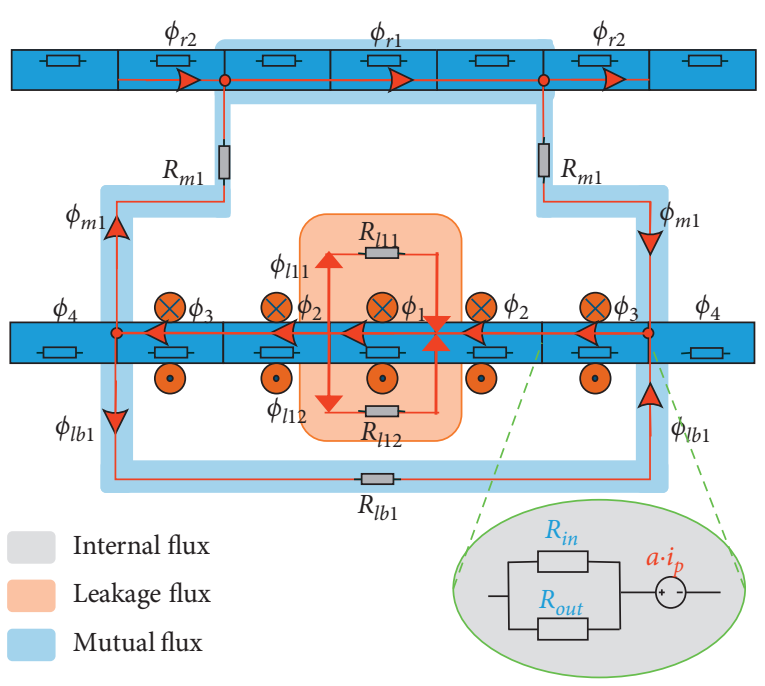

Figure 5: Equivalent magnetic reluctance network.

A lumped magnetic reluctance model was built in Figure 6, concerning voltage source and reluctance inside the core and in the air.

In the situation of uneven flux distribution, values of parameters $\left\{R_{11}, R_{12}, R_{13}, R_{14}\right\}$ and $\left\{R_{\mathrm{m} 1}, R_{\mathrm{m} 2}, R_{\mathrm{m} 3}, R_{\mathrm{m} 4}\right\}$ cannot be derived using the empirical equation. FEM simulation is applied once to obtain flux distribution $\left\{\phi_{1}, \phi_{2}\right.$, $\left.\phi_{3}, \phi_{4}\right\}$. Substitute the flux values into the model in Figure 6; the magnetic reluctance values can be derived according to Kirchhoff's voltage law.

In addition, the magnetic reluctance $R_{c}$ can be calculated as

$$
R_{c}=\frac{l_{c}}{\mu_{0} \mu_{r} A_{c}},
$$

where $l_{c}$ is the length of each individual core, $\mu_{0}$ is the vacuum permeability, and $\mu_{r}$ is the relative permeability of cores.

The total flux density along $y$-axis is

$$
B=\frac{\phi}{A_{c}},
$$

where $A_{c}$ is the cross-sectional area of the core in the $x-z$ plane.

After obtaining all of the parameters in the lumped magnetic reluctance model, the magnetic flux density under different working conditions can be acquired:

$$
B=\frac{A^{-1} U}{A_{c}},
$$

where $U$ is the magnetic motive force (MMF) matrix and $A$ is the magnetic reluctance coefficient.

It is worth noting that the flux density in the primary core is excited not only by the primary winding but also by the secondary winding. By combining the results generated by primary and secondary excitations, the flux density distribution in the primary core can be calculated.

As a result, for cores under sinusoidal current excitation, the magnetic loss can be calculated using the Steinmetz equation:

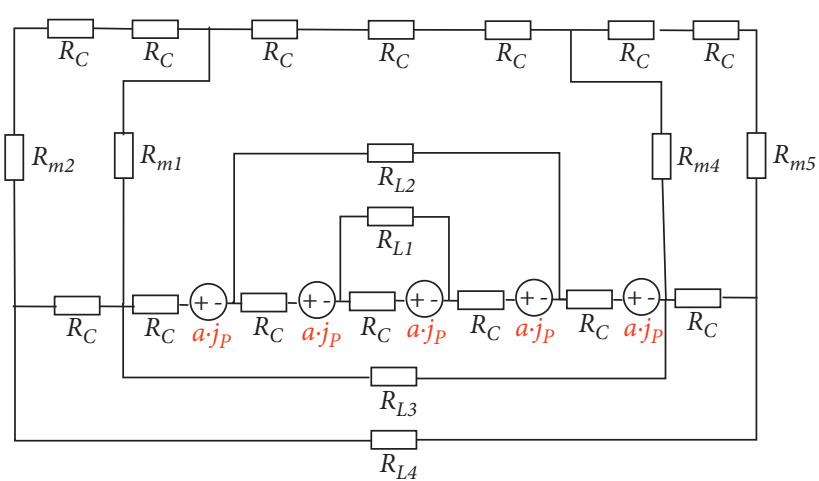

Figure 6: Lumped magnetic reluctance model.

$$
P_{V}=k \cdot f^{\alpha} \cdot \widehat{B}^{\beta},
$$

where $\widehat{B}$ is the peak induction of a sinusoidal excitation with frequency $f, P_{V}$ is the time-average power loss per unit volume, and $k$ and $\alpha$ are material parameters which can be obtained from the material datasheet.

By substituting (3) into (4), the magnetic loss in each section can be calculated. Generally, the highest flux density is designed well under saturation; thus, the ferrite usually works in the linear region and the overall magnetic loss can be summed.

\section{Influence of Winding Parameters}

To investigate the influence of winding parameters, we constructed two coupled coils, each with the same number of turns in total and different winding spaces between each turn. The receiver coil is designed to be homogeneous winding, while the transmitter coil was constructed with different spaces between each turn.

It would be desirable to reduce the magnetic loss while maintaining tight and compact windings. In this paper, different coil winding width ratio combinations were investigated and compared, in terms of coupling coefficient and magnetic loss.

The system configurations are as follows. The magnetic core is made of ultra-low-loss soft magnetic material DMR47. The overall dimension of the core is $500 * 380 * 12 \mathrm{~mm}$, which is formed by small magnetic cubes $(50 * 38 * 6 \mathrm{~mm})$. The number of primary and secondary coil turns is both 45 turns. The air gap between primary and secondary coils is $200 \mathrm{~mm}$. The system works at its resonant frequency $50 \mathrm{kHz}$. The input voltage is $160 \mathrm{~V}$ and the load of the system is $50 \Omega$.

The coils were equally divided into 5 portions. The number of portions determines the number of combination possibilities of winding density. With more portions, we can obtain a much more accurate result, but the calculation complexity will increase dramatically. The discretization number is a trade-off between precision and complexity. The current in each section $\alpha \cdot i_{p}$ corresponds to the number of turns. Serially connect the 5 portions, and calculate the coupling coefficient of the receiver side with the transmitter side. Apply different current in each portion to realize the 


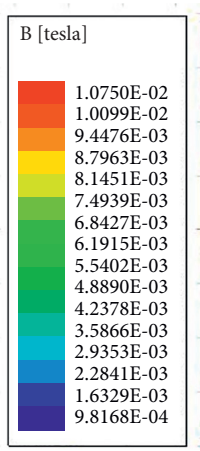

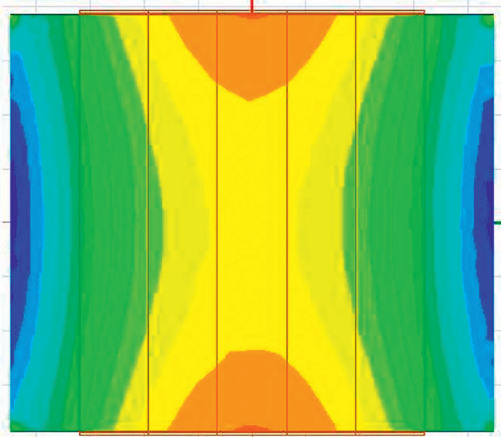

(a)
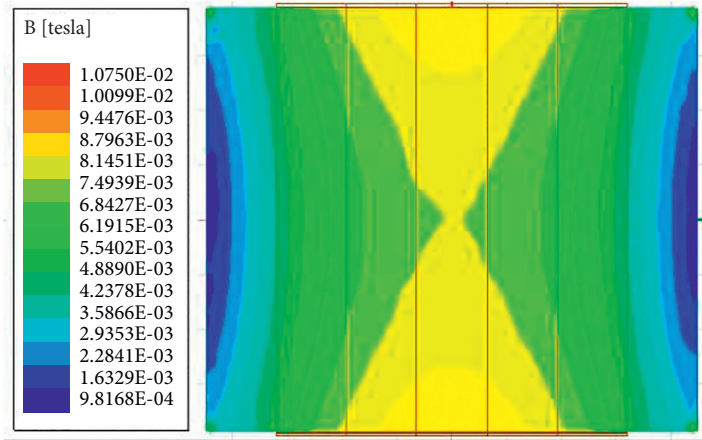

(b)
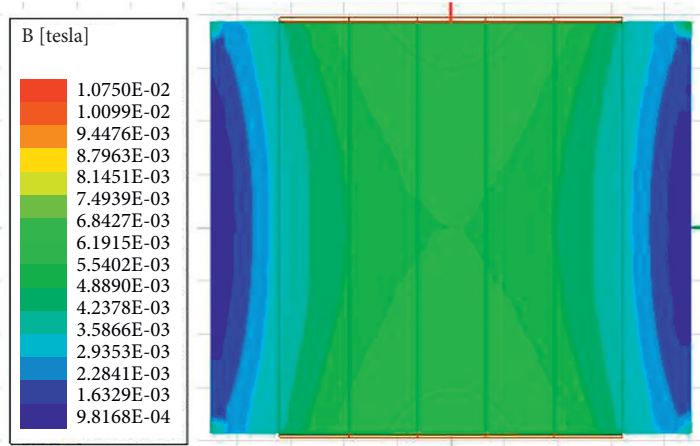

(c)

Figure 7: Magnetic field distributions in core with different winding parameters. (a) Homogeneous winding (9:9:9:9:9). (b) Winding parameters $(10: 10: 5: 10: 10)$. (c) Winding parameters $(17: 4: 3: 4: 17)$.

effects of inhomogeneous winding. A one-row five-column array indicates the current value in 5 portions. As seen in Figure 7, (9:9:9:9:9) indicates 9 turns in each portion.

The receiver coil remains homogeneous winding, while different winding parameters of the transmitter coil were studied to seek for the optimal combination. Typical theoretical results were compared with simulations in ANSYS Maxwell; the effects of inhomogeneous winding were shown in Figures 7(a)-7(c) under the same magnetic induction intensity scale. The simulation results agree well with theoretical analysis, as shown in Table 1.

In order to illustrate the effects of inhomogeneous winding on magnetic loss, the variations of coupling coefficient and core loss are depicted with different winding parameters, as shown in Figure 8.

The horizontal axis represents the coupling coefficient, while the vertical axis is $1 /$ core loss. The desired winding parameters are with high coupling coefficients and low core losses, so points positioned in the top right region are preferred.

As seen from Figure 8, the range of coupling coefficient is limited between 0.118 and 0.122 . Homogeneous winding ( 9 , $9,9,9,9)$ has the worst performance, with the lowest coupling coefficient and highest core loss, compared with other cases with coarse winding in center. Inhomogeneous winding effectively reduced the magnetic loss in the ferrite core. The optimal situation within consideration range, with winding parameter $(17,4,3,4,17)$, reduced the core loss by $5.6 \%$ compared with the homogeneous case, while the coupling coefficient increased by $1.9 \%$. When designing the
TABLE 1: Comparison of core loss in theoretical and simulation results.

\begin{tabular}{lcc}
\hline Winding parameters & Theoretical loss $(W)$ & Simulation loss $(W)$ \\
\hline$(9: 9: 9: 9: 9)$ & 16.05 & 16.08 \\
$(10: 10: 5: 10: 10)$ & 15.55 & 15.58 \\
$(17: 4: 3: 4: 17)$ & 15.10 & 15.12 \\
\hline
\end{tabular}

winding parameters, relative low magnetic field density in the core center helps to reduce the overall core loss.

\section{Experimental Result and Discussion}

Measurements for wireless power transfer system under inhomogeneous winding were obtained to evaluate whether the power loss reduction found for inhomogeneous winding translated to improve power efficiency well.

Primary coil turns are winded around the core with different spaces between each turn. The total number of turns remains constant, and the working frequency is unchanged, so the copper loss is assumed to be the same, and so is the eddy-current loss. The circuit always works at the soft switching mode, so the switching loss remain unchanged. Therefore, with different winding parameters, the variation in core loss results in the change in system efficiency. The overview of the experiment system is shown in Figure 9.

The diagram of setups for measuring is shown in Figure 10. Test waveforms of the system are shown in Figure 11, including the primary current $i_{p}$, secondary current $i_{s}$, and 


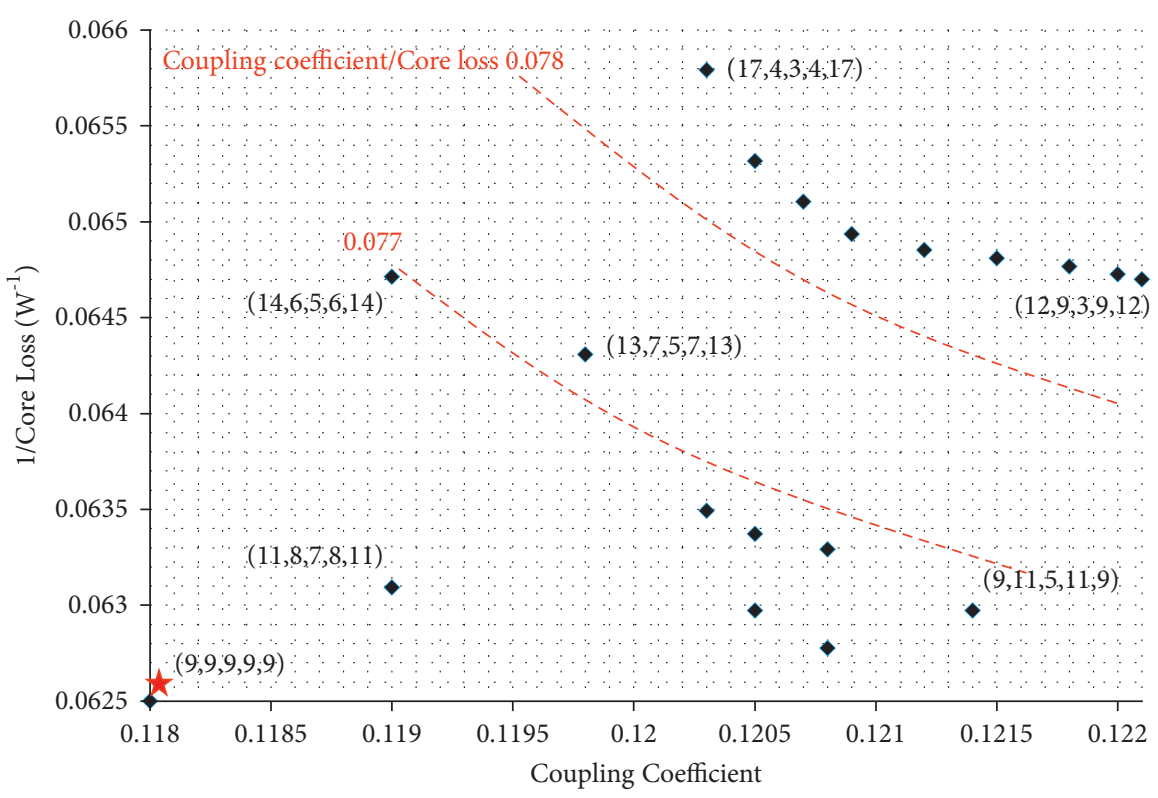

FIgURE 8: Variation of core loss and coupling coefficient with different winding parameters.

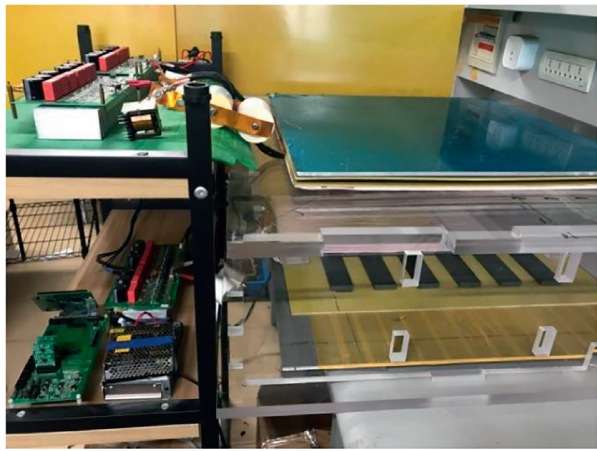

FIGURE 9: Experimental setup for wireless power transfer under inhomogeneous winding.

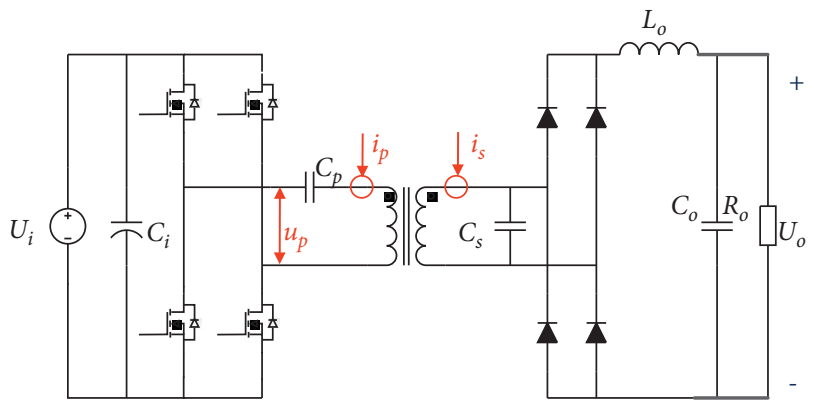

FIgURE 10: The diagram of the setups for measuring.

output voltage of the inverter $u_{p} . u_{g}$ represent the gate driving signal for MOSFET in the inverter bridge. The system works under ZVS condition, as not much of a voltage spike is observed in the waveform.

The experimental and calculation results are compared in Figure 12. Since it is difficult to directly obtain the core loss, the experimental core losses were obtained by subtracting the measured total losses with the measured

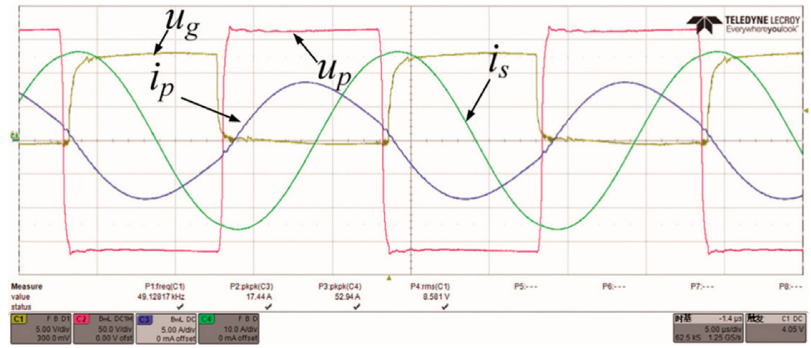

Figure 11: Test waveforms of the system.

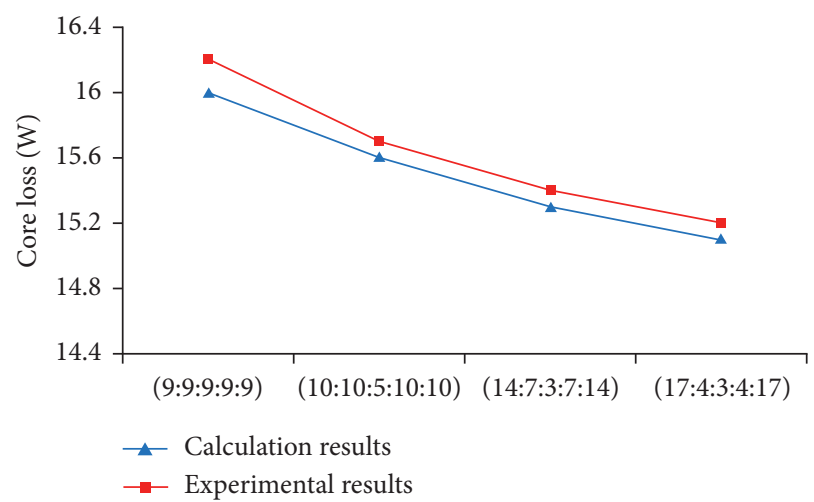

FIgURE 12: Variation of core loss with different winding parameters.

winding losses by using a LCR meter, the switching device losses, and the diode losses by integrating the voltage and current waveforms using a HDO4034 oscilloscope. The variation of measured core loss agrees well with the calculated results, assuming the winding loss and switching loss remain unchanged under the same frequency. The system power loss significantly reduces as coils are wound relatively coarsely in the center and tightly at two ends. 


\section{Conclusions}

A novel inhomogeneous winding method for loosely coupled transformers is proposed in the paper. A magnetic reluctance model for solenoid structure is built to calculate the core loss under inhomogeneous winding. Sweeping maps with different primary winding parameters were provided to investigate the optimal combination. The obtained experimental results show great agreement with the presented optimization. Compared with traditional homogeneous winding, the new inhomogeneous winding method effectively reduces magnetic loss in the ferrite core, while maintaining tight coupling between primary and secondary coils.

\section{Data Availability}

The experimental data used to support the findings of this study are included within the article.

\section{Conflicts of Interest}

The authors declare that they have no conflicts of interest.

\section{Acknowledgments}

The authors acknowledge the funding of Zhejiang Key R\&D Program, under Grant no. 2019C01044.

\section{References}

[1] S. Lee, D. H. Kim, Y. Cho et al., "Low leakage electromagnetic field level and high efficiency using a novel hybrid loop-array design for wireless high power transfer system," IEEE Transactions on Industrial Electronics, vol. 66, no. 6, pp. 4356-4367, 2019.

[2] M. Y. Li, X. Y. Chen, H. Y. Gou et al., "Conceptual design and characteristic analysis of a sliding-type superconducting wireless power transfer system using ReBCO primary at $50 \mathrm{~Hz}$," IEEE Transactions on Applied Superconductivity, vol. 29, no. 2, Article ID 5501304, 2019.

[3] Z. H. Ye, Y. Sun, X. Dai, and C. Tang, "Energy efficiency analysis of U-coil wireless power transfer system," IEEE Transactions on Power Electonics, vol. 31, no. 7, pp. 4809-4817, 2019.

[4] Z. Yan, Y. Li, C. Zhang, and Q. Yang, "Influence factors analysis and improvement method on efficiency of wireless power transfer via coupled magnetic resonance," IEEE Transactions on Magnetics, vol. 50, no. 4, Article ID 4004204, 2014.

[5] J. Liu, Q. Deng, D. Czarkowski, M. K. Kazimierczuk, H. Zhou, and $\mathrm{W}$. Hu, "Frequency optimization for inductive power transfer based on AC resistance evaluation in litz-wire coil," IEEE Transactions on Power Electronics, vol. 34, no. 3, pp. 2355-2363, 2019.

[6] Z. Cheng, Y. Lei, K. Song, and C. Zhu, "Design and loss analysis of loosely coupled transformer for an underwater high-power inductive power transfer system," IEEE Transactions on Magnetics, vol. 51, no. 7, Article ID 8401110, 2017.

[7] X. Liu, C. Liu, W. Han, and P. W. T. Pong, "Design and implementation of a multi-purpose TMR sensor matrix for wireless electric vehicle charging," IEEE Sensors Journal, vol. 19, no. 5, pp. 1683-1692, 2019.

[8] T. Gonda, S. Mototani, K. Doki, and A. Torii, "Effect of air space in waterproof sealed case containing transmitter and receiver of wireless power transfer in sea water," Electrical Engineering in Japan, vol. 206, pp. 24-31, 2019.

[9] J. Muhlethaler, J. Biela, J. W. Kolar, and A. Ecklebe, "Core losses under the DC bias condition basedon steinmetz parameters," IEEE Transactions on Power Electronics, vol. 27, no. 2, pp. 953-963, 2012.

[10] S. C. Tang and N. J. McDannold, "Power Loss analysis and comparison of segmented and unsegmented energy coupling coils for wireless energy transfer," IEEE Journal of Emerging and Selected Topics in Power Electronics, vol. 3, no. 1, pp. 215-225, 2015.

[11] K. E. I. Elnail, X. L. Huang, X. Chen, L. L. Tan, and H. Z. Xu, "Core Structure and electromagnetic field evaluation in WPT systems for charging electric vehicles," Energies, vol. 11, no. 7, p. 1734, 2018.

[12] X. Zhang, S. L. Ho, and W. N. Fu, "Quantitative analysis of a wireless power transfer cell with planar spiral structures," IEEE Transactions on Magnetics, vol. 47, no. 10, pp. 32003203, 2011.

[13] Y. Tang, F. Zhu, and H. Ma, "Efficiency optimization with a novel magnetic-circuit model for inductive power transfer in EVs," Journal of Power Electronics, vol. 18, no. 1, pp. 309-322, 2018. 